\title{
EL FENOMENO EL NIÑO 1992-93: SU INFLUENCIA EN LA BIOLOGIA REPRODUCTIVA DE Tagelus dombeii
}

\author{
Victor Ishiyama* y Betty Shiga*
}

\begin{abstract}
RESUMEN
El fenómeno El Niño 1992-93, indujo un incremento en la cantidad de ejemplares maduros y en desove de gametos de Tagelus dombeii, debido al aumento de la temperatura superficial del mar. La proporción de sexos fue de 1,2:1,0 a favor de las hembras. En la gametogénesis acelerada, se diferenció: ovogonias y ovocitos (en previtelogénesis) ovocitos I (en vitelogénesis primario) y ovocitos II (en vitelogénesis secundario). La escala de madurez gonadal, con altos porcentajes de ejemplares maduros y en desove, y pocos inmaduros y en desarrollo, se clasificó en: indiferenciados, inmaduros, en desarrollo, maduros y desovados.
\end{abstract}

\begin{abstract}
The EI Niño 1992-93 phenomena incremented the number of matures males and females, and induced the spawning of Tagelus dombeil, due to the rise of the sea water temperature. The sex proportion was $1.2: 1.0$, (female:male). In the accelerated gametogenesis, we differentiated: oogonias and oocytes (previtelogenesis), ovocyles I (primary vitelogenesis) and ovocytes II (secondary vitelogenesis). The maturity scale, with high percentage of matures specimens and in spawning, and scarce immaturity and in development, were classificated in : non differentiated, immaturity, mature and spawn.
\end{abstract}

Palabras clave: Reproducción, Tagelus dombeil, Solercutidae.

\section{INTRODUCCION}

Durante 1992-93, se presentó el fenómeno El Niño, que se caracterizó por la inestabilidad de las condiciones atmosféricas y oceánicas que generó ondas de calor, con máximo en los meses de febrero, junio y agosto de 1993, para ir declinando paulatinamente.

El fenómeno El Niño influye en forma distinta en los diferentes niveles del ecosistema, lo que se refleja en el comportamiento de las especies y sus pesquerías; algunos invertebrados marinos son afectados, produciendo mortandad; en otras induce a un incremento acelerado de la madurez gonadal, que se manifiesta en porcentajes elevados de ejemplares en evacuación de gametos, y en otros se produce una mortandad para luego recuperar su equilibrio.
La especie más estudiada durante el fenómeno El Niño 1982-83 fue la concha de abanico Argopecten purpuratus, de la Bahía de Independencia, Pisco y de la Zona del Callao, Lima, trabajos que fueron realizados por Wolff, (1984, 1985 y 1988); Valdivieso \& Alarcón (1985); Mejía et. al. (1985), Mendo et. al. (1988), Vargas et. al. (1988); Yamashiro \& Mendo (1988) Chávez \& Ishiyama (1989), Yamashiro \& Espino (1991). También se realizaron estudios sobre mitílidos en la Bahía de Independencia, Pisco y en la Bahía de Ancón, Lima (Soenes, 1985 ; Arntz \& Valdivia, 1985 ; Tarazona et al., 1988).

Los estudios sobre la influencia que pueda ejercer el fenómeno El Niño en el comportamiento reproductivo de otras especies de moluscos que tienen importancia económica para el país es de vital importancia para poder comprender los cambios que ocurren y

* Laboratorio de Reproducción y Biología del Desarrollo. Facultad de Ciencias Biológicas, Ĺniversidad Nacional Mayor de San Marcos 
Tabla 1. Proporción de sexos de Tagelus dombeii

\begin{tabular}{|c|c|c|c|}
\hline MESES & $\begin{array}{c}\text { HEMBRAS } \\
N(\%)\end{array}$ & $\begin{array}{c}\text { MACHOS } \\
N(\%)\end{array}$ & $\begin{array}{c}\text { RELACIÓN } \\
\mathrm{H}: \mathrm{M}\end{array}$ \\
\hline My -92 & $11(47,8)$ & $12(52,20)$ & $1,0: 1,0$ \\
\hline $\ln$ & $13(48,1)$ & $14 \quad(5 I, 9)$ & $1,0: 1,0$ \\
\hline Jl & $09 \quad(50,0)$ & $09(50,0)$ & $1,0: 1,0$ \\
\hline A & $17(58,6)$ & $12 \quad(41,4)$ & $1,4: 1,0$ \\
\hline S & $13(61,9)$ & $08 \quad(38,1)$ & $1,6: 1,0$ \\
\hline o & $12(54,5)$ & $10 \quad(45,5)$ & $1,2: 1,0$ \\
\hline $\mathrm{N}$ & $14 \quad(58,3)$ & $10(41,7)$ & $1,4: 1,0$ \\
\hline D & $15 \quad(51,7)$ & $14 \quad(48,3)$ & $1,0: 1,0$ \\
\hline E-93 & $21 \quad(75,0)$ & $07 \quad(25,0)$ & $3,0: 1,0$ \\
\hline$F$ & $15(56,7)$ & $12(44,3)$ & $1,2: 1,0$ \\
\hline$M$ & $16(45,7)$ & $19(54,3)$ & $1,0: 1,2$ \\
\hline A & $18 \quad(47,4)$ & $20 \quad(52,6)$ & $1,0: 1,2$ \\
\hline My & $23 \quad(62,2)$ & $14 \quad(37,8)$ & $1,6: 1,0$ \\
\hline In & $19(48,7)$ & $20 \quad(51,3)$ & $1,0: 1,0$ \\
\hline $\mathrm{Jl}$ & $25 \quad(62,5)$ & $15 \quad(37,5)$ & $1,6: 1,0$ \\
\hline A & $16(47,0)$ & $18 \quad(53,0)$ & $1,0: 1,2$ \\
\hline$S$ & $15 \quad(38,5)$ & $24 \quad(61,5)$ & $1,0: 1,6$ \\
\hline $\mathrm{O}$ & $22(64,7)$ & $12 \quad(35,3)$ & $1,8: 1,0$ \\
\hline TOTAL & $294(54,0)$ & $250(46,0)$ & $1,2: 1,0$ \\
\hline
\end{tabular}

tenerlos presentes para otros fenómenos El Niño que ocurrirán en el futuro.

Esto indujo a trabajar con el bivalvo "concha navaja" Tagelus dombeii de la zona de San Bartolo, Lima, durante el fenómeno El Niño 1992-93, para obtener información sobre el comportamiento reproductivo de la especie y comparar los resultados con los obtenidos en condiciones oceanográficas normales.

\section{MATERIALES Y METODOS}

De mayo de 1992 a octubre de 1993, en la playa de San Bartolo (12 $\left.23^{\prime} \mathrm{S}, 76^{\circ} 47^{\prime} 0\right)$ se realizó la recolección al azar de un total de 544 ejemplares de $\mathcal{T}$. dombeii con un promedio mensual de 30 individuos. En el laboratorio se tomaron las mediciones morfométricas de cada ejemplar, para luego extraer las gónadas y fijarlos en formol al $10 \%$ en agua de mar; posteriormente se les incluyó en Paraplast.

Los cortes histológicos de $7 \mu \mathrm{m}$. de grosor, se deshidrataron y las láminas histológicas se colorearon con Hematoxilina-Eosina, esto nos permitió tener una secuencia de los fenómenos que están ocurriendo durante la madurez gonadal, durante el ciclo reproductivo anual. Las observaciones morfológicas y el análisis histológico nos han permitido encontrar resultados sobre la primera madurez, la gametogénesis, madurez gonadal y el comportamiento reproductivo en general.

\section{RESULTADOS}

\section{Características morfológicas.}

T. dombeii se distribuye desde Panamá a Valdivia en Chile (Alamo \& Valdivieso, 1987) ; como en la mayoría de los moluscos, no se observa dimorfismo sexual, la gónada se localiza en la masa visceral del animal ; su tamaño y color varía de acuerdo a la madurez gonadal, en los machos cambia del color crema (inmaduro) al lechoso (maduro) y en las hembras del color crema (inmaduro) al marrón (maduro).

La estructura histológica de la gónada, presenta un epitelio cúbico simple, seguido del tejido conectivo denso irregular, cuyas fibras colágenas, con las fibras musculares, se disponen en forma circular, longitudinal y oblicua formando una capa muscular gruesa que de trecho en trecho se introduce en los espacios que quedan entre los folículos o túbulos seminiferos. El tubo digestivo atraviesa la gónada en toda su longitud (fig. 6a, b, f).

Los datos sobre la relación de sexos fue de 1,2:1,0 a favor de las hembras (tabla 1). $\mathrm{El}$ ancho de $T$. dombeii que fueron colectados al azar, fue de 41,7 a $90,4 \mathrm{~mm}$, con un promedio de $73,8 \mathrm{~mm}$, (los machos de 42,3 a $90,4 \mathrm{~mm}$. y las hembras de 41,7 a $90,0 \mathrm{~mm}$.) (fig. 1 y 2): la mayor cantidad de ejemplares se encontraron de 64.0 a $79.0 \mathrm{~mm}$ (fig. 1) y el promedio del ancho mínimo fue de $54,5 \mathrm{~mm}$ 


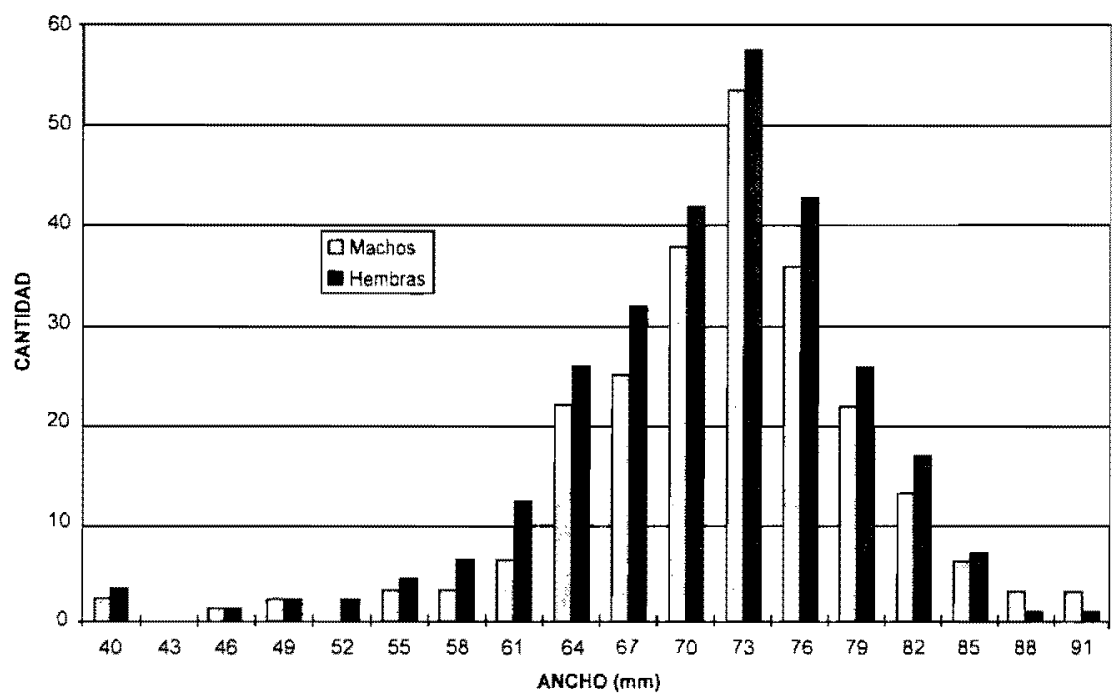

Fig. 1.- Tamaño del ancho de Tagelus dombeii, colectados de mayo del 92 a octubre del 93

y el máximo de $86,0 \mathrm{~mm}$. (fig. 3).

Durante el fenómeno El Niño 1992-93 fue difícil determinar la talla mínima de primera madurez real, debido al incremento acelerado de ejemplares maduros y en desove, en estas condiciones, se observó a las hembras a partir de los $54 \mathrm{~mm}$ y a los machos de los 59 $\mathrm{mm}$ del ancho de la valva, estos datos son de suma importancia para determinar el potencial reproductivo de la especie.

Mientras que la longitud de los animales fue de 21 a $30 \mathrm{~mm}$ con un promedio de 23,4 $\mathrm{mm}$ (los machos de 21 a $25 \mathrm{~mm}=$ promedio
$23 \mathrm{~mm}$ y las hembras de 21,9 a $30,8 \mathrm{~mm}=$ promedio $23.8 \mathrm{~mm}$ )(fig. 4). El peso total del animal (valvas + visceras) fue de 16,6 a 27,1 g con un promedio de $21,0 \mathrm{~g}$ y el peso de las vísceras o partes blandas del animal ( $\sin$ las valvas), fue de $7,5 \mathrm{~g}$ a $15,2 \mathrm{~g}$ con un promedio de $10,6 \mathrm{~g}$, siendo evidente que el $50 \%$ del peso total del animal corresponde a las valvas (fig. 5), El peso total del animal y el peso de las partes blandas (vísceras) del animal fue mayor en junio, de setiembre (1992) a marzo (1993), en junio y en octubre (1993), coinci-

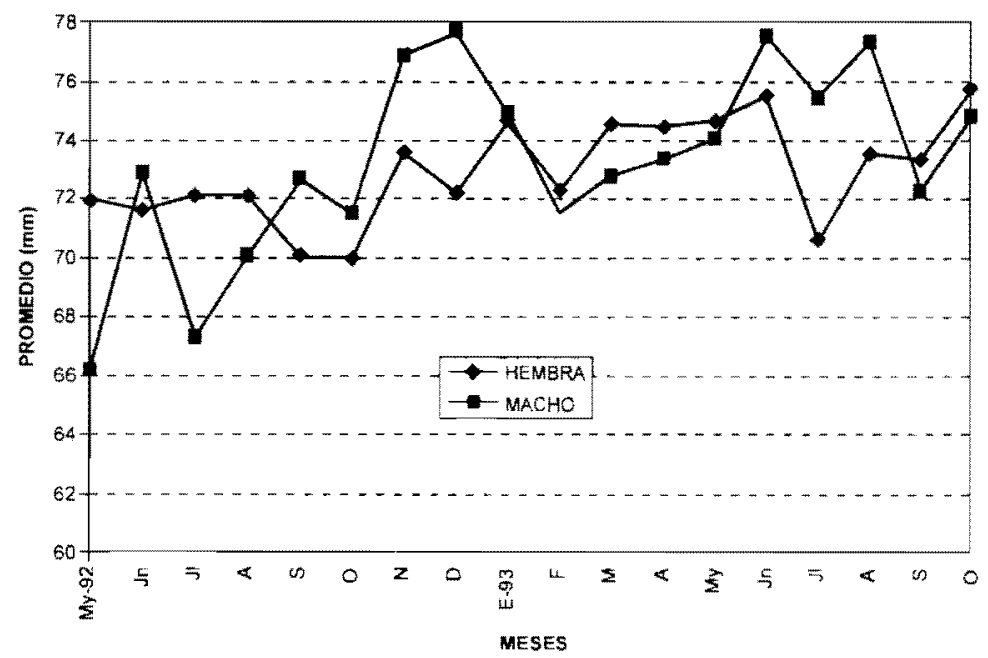

Fig. 2.- Promedio mensual del ancho de Tagelus dombeii 


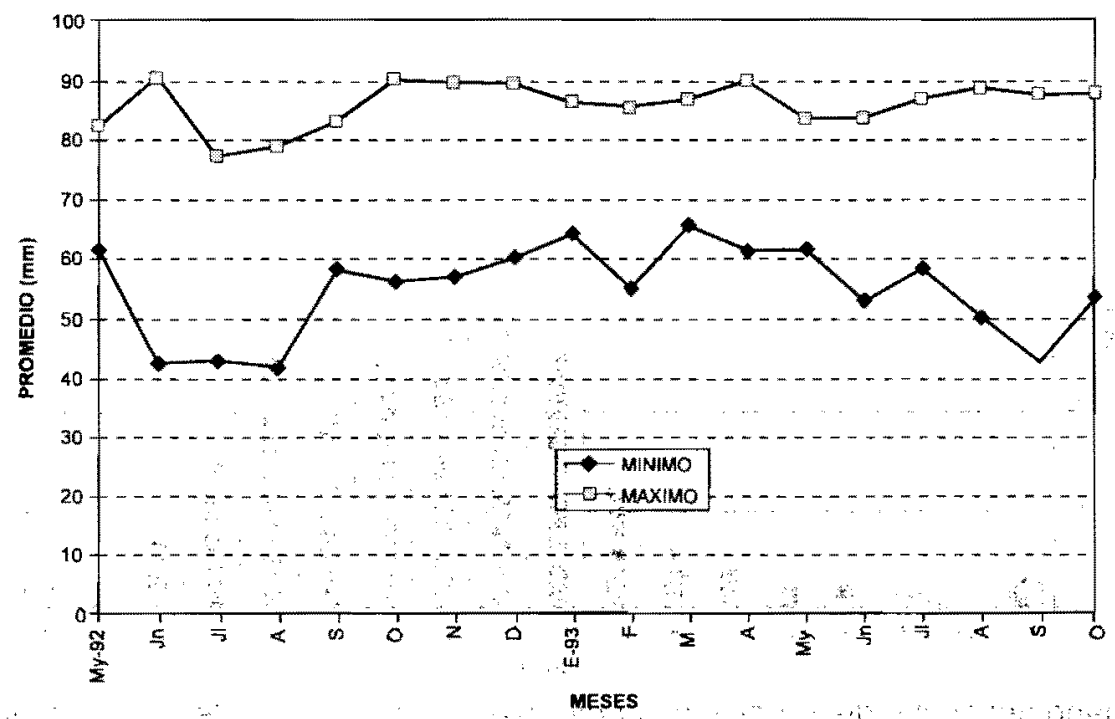

Fig. 3.- Promedio mensual del ancho mínimo y máximo de Tagelus dombeil

diendo con la máxima madurez de $T$. dombeii, y los menores pesos, en julio (1992), de enero a mayo, en julio y en setiembre (1993), con el desove de gametos (fig. 5 y 8 ).

Ovogénesis.- De acuerdo a la rápida diferenciación de los gametos, se pudieron determinar las siguientes características :

Ovogonias, muy pequeñas, fusiformes o esféricas, el núcleo ocupa casi toda la célula, se encuentran adheridas a la pared del folículo, tienen un promedio de $18 \mu \mathrm{m}$ de diámetro.

Ovocito de I orden, donde se inicia la síntesis del vitelo (previtelogénesis), las células van aumentando de tamaño, tomando la forma ovalada a cuadrangular, con un promedio de $55 \mu \mathrm{m}$ de diámetro; luego los ovocitos adquieren la forma rectangular hasta la forma de una gota de agua (vitelogénesis primaria), están adheridos a la pared del folículo, tienen un promedio de $85 \times 102 \mu \mathrm{m}$.

Ovocitos de II orden, (vitelogénesis secundaria) los gránulos del vitelo llenan el citoplasma, adoptan la forma esférica y quedan libres en la cavidad del folículo o toman la forma poliédrica por la presión que ejercen unos contra otros al llenar los folículos tienen un promedio de $95 \mu \mathrm{m}_{\text {s }}$ listos para ser desovados.

Escala de madurez gonadal.- Los procesos de maduración de la gónada se determinaron por el estudio histólógico secuencial, durante todos los meses que duró el estudio y que fueron complementados con las observaciones morfológicas de las gónadas, esto nos permitió determinar cinco estadíos de madurez gonadal:

Indiferenciado, la gónada flácida, con los folículos y túbulos seminíferos muy pequeños, no se puede diferenciar el sexo del animal (fig. 7a) : están considerados los juveniles que no han llegado a la primera madurez y los que han realizado la evacuación total de gametos.

Inmaduros, en las hembras, los folículos comienzan a crecer por la proliferación de -ovogonias y ovocitos, que se encuentran adheridos a la pared del folículo (fig. 6a). En los machos, los túbulos seminíferos pequeños con espermatogonias y espermatocitos (fig. 7b). 


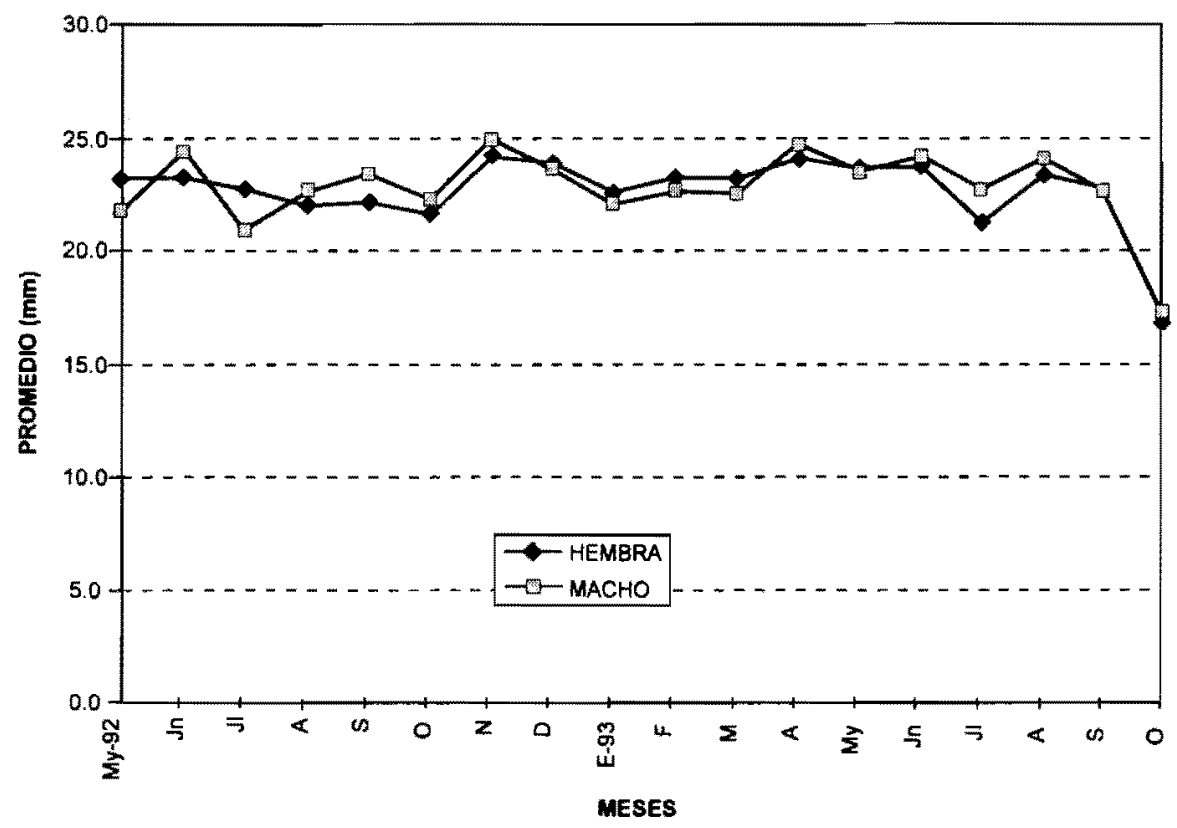

Fig. 4.- Promedio mensual de la longitud (mm) de Tagelus dombeii

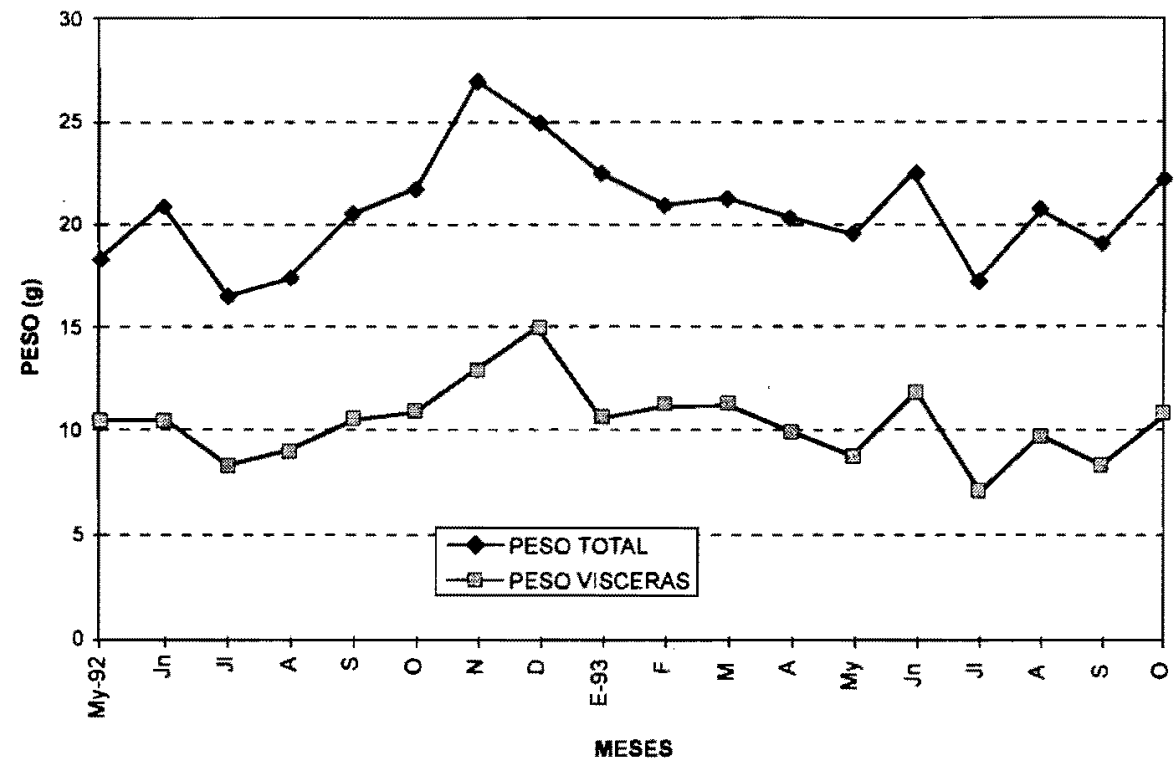

Fig. 5.- Promedio del peso total y del peso de las vísceras de Tagelus dombeii 

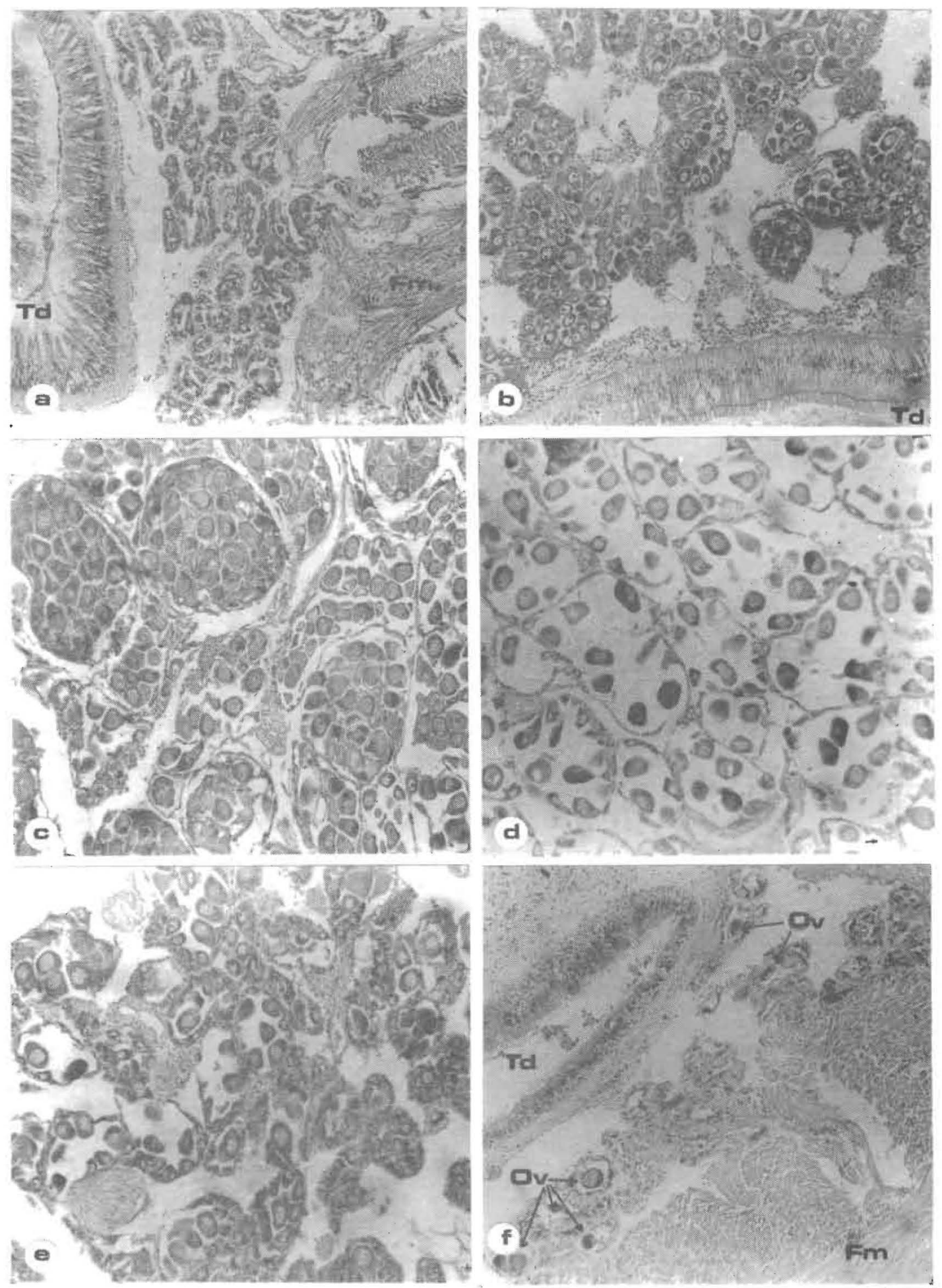

Fig. 6.- Escala de madurez gonadal en las hembras de Tagelus dombeii: a) inmaduro, b) en desarrollo, c) maduro, d) inicio evacuación, e) evacuación parcial, f) evacuación total. Td = Tubo digestivo, $\mathrm{Ov}=$ Ovocito atrésico, $\mathrm{Fm}=$ Fibras musculares. Coloración $\mathrm{H}-\mathrm{E}, 100 \mathrm{x}$. 

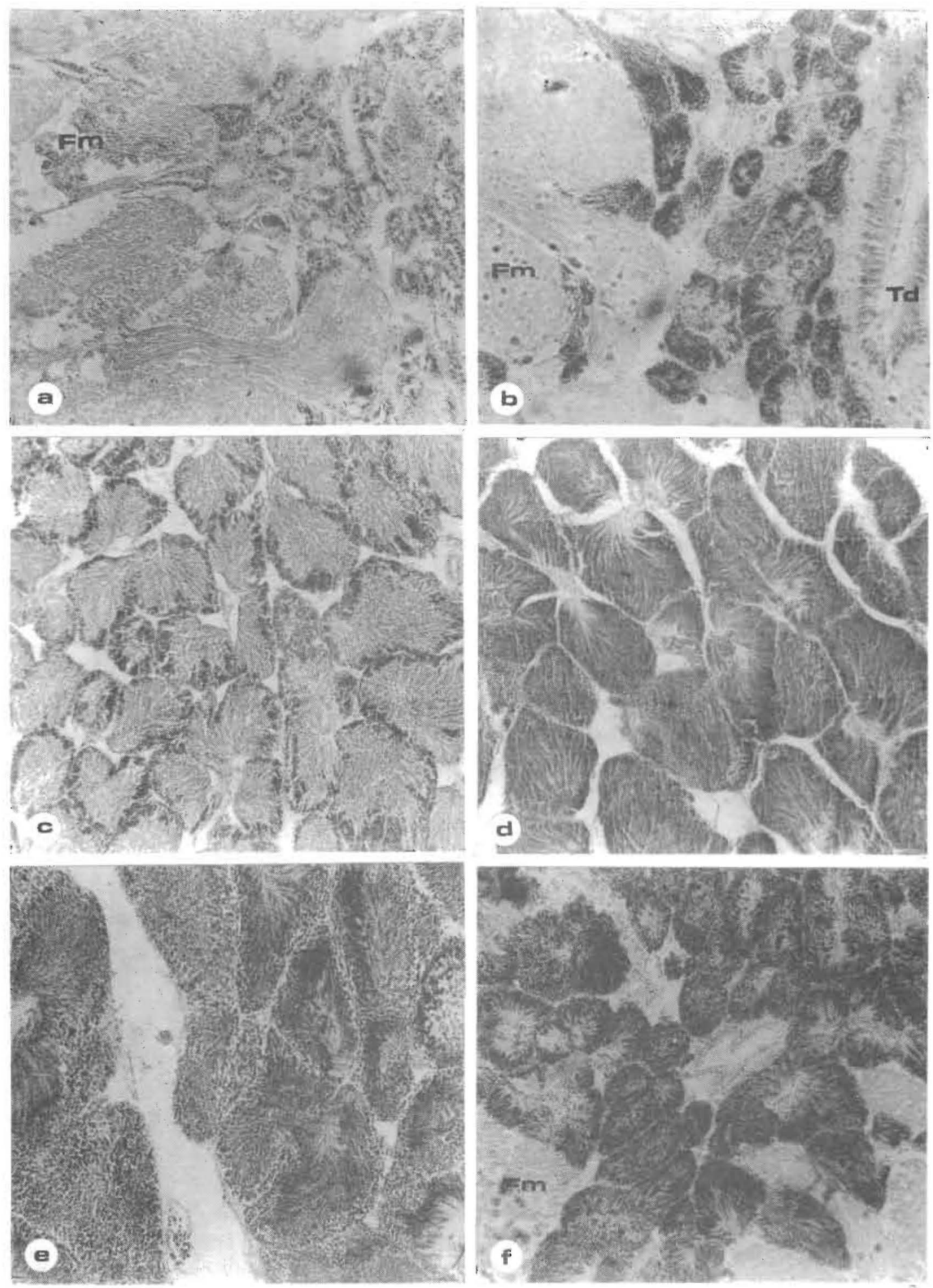

Fig. 7.- Escala de madurez gonadal en los machos de Tagelus dombeii: a) indiferenciado, b) inmaduro, c) en desarrollo, d) maduro, e) inicio evacuación, f) evacuación. Td = Tubo digestivo, Fm = Fibras musculares. Coloración H-E, 100x. 


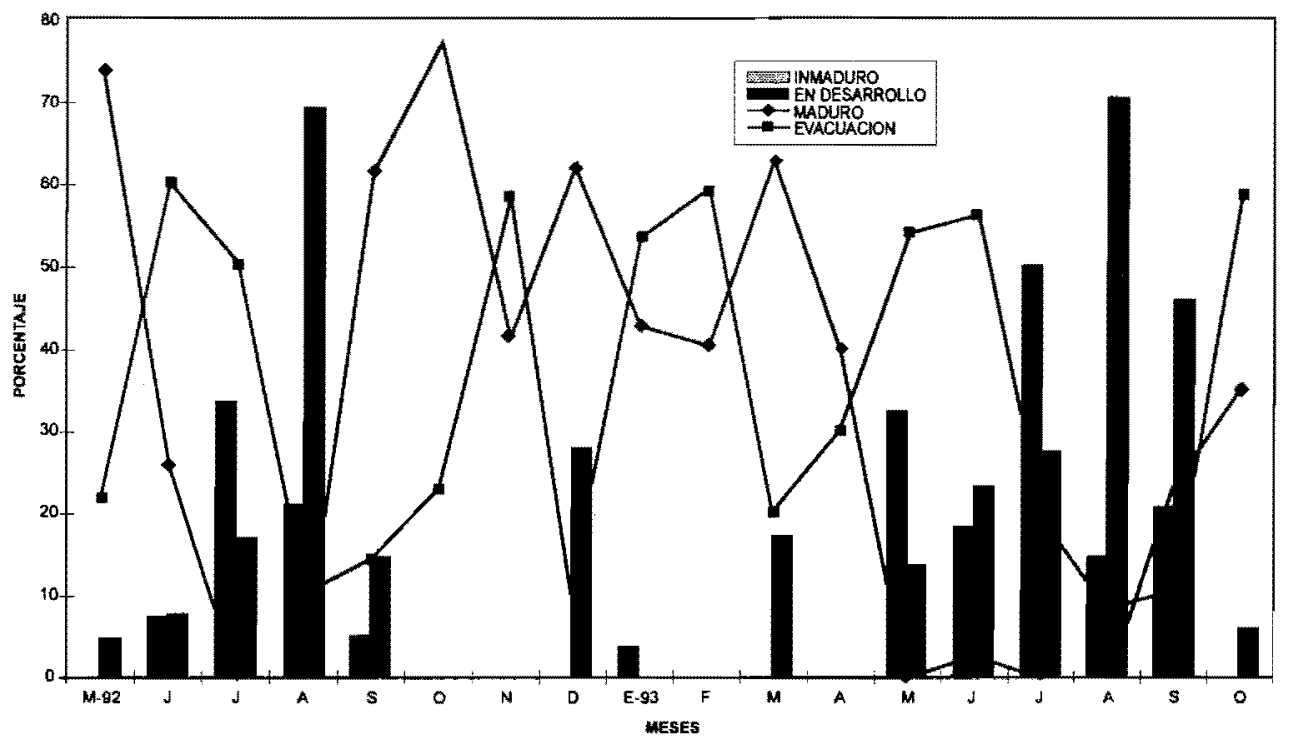

Fig. 8.- Porcentaje de los estadios de madurez gonadal de Tagelus dombeii.

En desarrollo, en las hembras, los ovocitos van tomando la forma elongada y se mantienen unidos a la pared del folículo por el pie o pedúnculo, algunos se desprenden y toman la forma esférica y quedan libres en la cavidad folicular (fig. 6b). En los machos, se observan espermatogonias, espermatocitos y algunos espermatozoides (fig. $7 \mathrm{c}$ ).

Maduros, en las hembras, los folículos grandes están llenos de ovocitos maduros, que se encuentran libres en la cavidad (fig. 6c). En los machos, los túbulos seminíferos bien desarrollados, con espermatogonias, espermatocitos y espermatozoides compactos que se disponen en forma radial hacia la luz del túbulo seminífero (fig. $7 \mathrm{~d}$ ).

Evacuación, la evacuación de los gametos en los machos y en las hembras se produce por emisiones alternadas; en una fase inicial, se observan espacios vacíos en los folículos y túbulos seminíferos, que se van haciendo más notorios, hasta que se produce la evacuación total de los gametos (fig. $6 \mathrm{~d}, \mathrm{e}, \mathrm{f}$ y 7 e,f).

\section{Reproducción}

Es evidente que el aumento de la temperatura superficial del mar, como consecuencia de el fenómeno El Niño 1992-93, provocó un incremento en la madurez gonadal de los ejemplares de T. dombeii. De mayo de 1992 a octubre de 1993 se observaron porcentajes altos de ejemplares maduros y en evacuación, debido a que los gametos comenzaron a madurar en forma acelerada.

En las hembras, la evacuación de gametos, del $20 \%$ en mayo de 1992, aumentó al 60 y $50 \%$ en junio y julio, respectivamente; descendió al $12 \%$ en agosto y fue incrementando paulatinamente hasta noviembre $(60 \%)$, disminuyó al $13 \%$ en diciembre, para incrementar nuevamente hasta 55 y $60 \%$ en enero y febrero de 1993, respectivamente, para descender al $22 \%$ en marzo y aumentar al $55 \%$ en mayo y $58 \%$ en junio, e ir disminuyendo al $20 \%$ en julio, $12 \%$ en agosto e ir aumentando hasta el $60 \%$ en octubre de 1993 . Los ejemplares en evacuación de gametos estaban precedidos de ejemplares maduros en porcenta= jes altos entre 75 al $80 \%$ (fig. 8). 


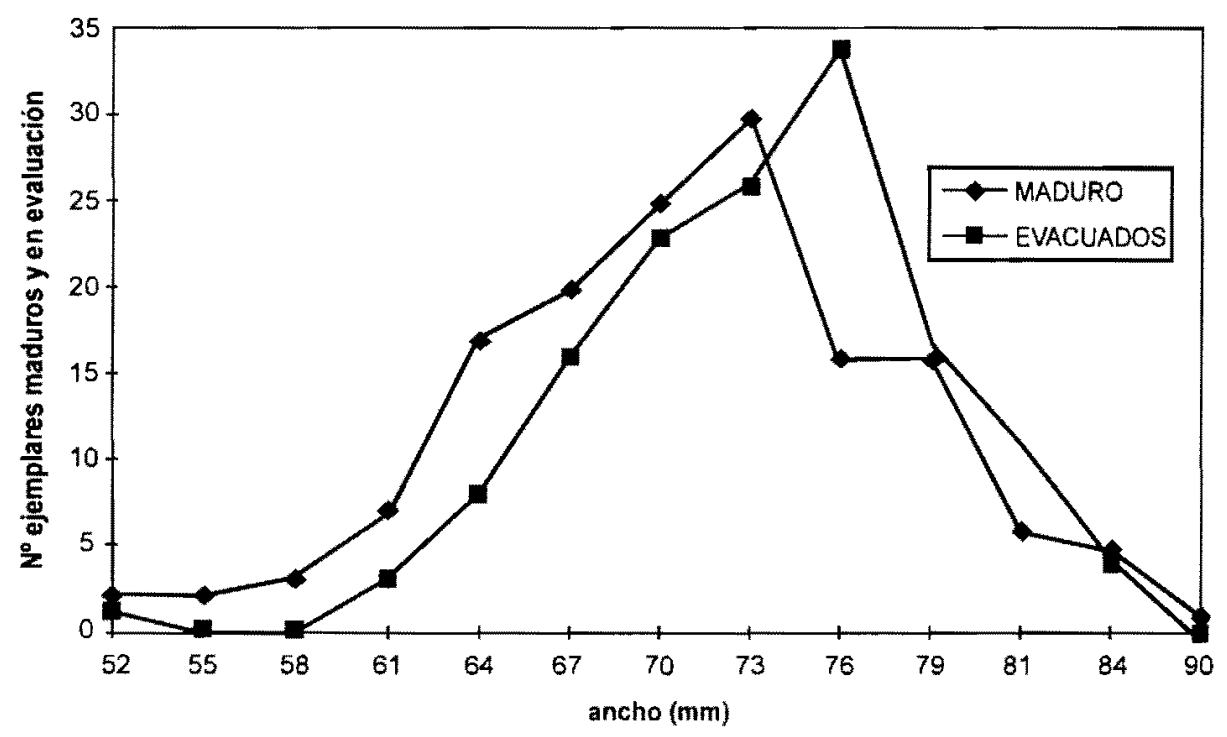

Fig. 9.- Tamaño del ancho $(\mathrm{mm})$ de Tagelus dombeii en ejemplares maduros y en evacuación de gametos.

Los ejemplares inmaduros y en desarrollo, se observaron en porcentajes bajos (fig. 8). La mayor cantidad de ejemplares maduros y en evacuación de gametos se encontraron entre 64 y $79 \mathrm{~mm}$ del ancho del animal (fig. 9).

\section{DISCUSION}

Con el fenómeno El Niño 1982-83, se comienzan a realizar estudios sobre la flora y fauna de nuestro litoral y las consecuencias positivas y negativas de las mismas; durante 1982-83, la especie más estudiada fue la concha de abanico Argopecten purpuratus, debido a la proliferación que se produjo como consecuencia del aumento de la temperatura del mar (Wolff, 1984a); durante el post El Niño se observaron porcentajes altos de ejemplares en desove, inicialmente en forma irregular, para ir incrementando en intensidad $y$, al estudiar los cortes histológicos de las gónadas, en las partes desovadas se producía un rápido desarrollo de la gametogénesis (Chavez \& Ishiyama, 1989 ; Ishiyama \& Shiga, 1997). Illanes et al.,(1985) describe que el aumento de la temperatura del mar a $21^{\circ} \mathrm{C}$ estimuló la evacuación de gametos, coincidiendo c̀n lo reportado por Loosanoff \& David (1952), Sartry (1963, 1966), Wolff (1988), Vargas et al. (1988), quienes observaron que el índice gonadosomático fue evidente cuando la temperatura del agua de mar llegó hasta $25^{\circ} \mathrm{C}$ y de $17^{\circ}$ a $20^{\circ} \mathrm{C}$, respectivamente; mientras que Samamé et al. (1985a, b, 1986), Mendo etal (1988), Chávez \& Ishiyama (1989) e Ishiyama \& Shiga (1997) sugieren que el aumento de las poblaciones de Argopecten purpuratus fue más intenso durante el post El Niño.

En otras especies de moluscos, sólo se reporta el trabajo en Aulacomya ater y Semymitilus algosus en la Bahía de Ancón y Bahía Independencia, donde se reporta que el fenómeno El Niño 1982-83 fue negativo para ambas especies, debido a que produjo una gran mortandad, para iniciar su recuperación en octubre de 1983 para ambos mitílidos de la Bahía de Ancón, y en agosto de 1986 en la Bahía Independencia (Soenes, 1985 ; Arntz \& Valdivia, 1985; Tarazona et al, 1988).

Durante el fenómeno El Niño $1992-93$ observamos que $T$. dombeii incrementó su producción de ejemplares maduros y en desove 
WOLFF, M. 1985. Abundancia masiva y crecimiento de pre-adulto de la concha de abanico (Argopecten purpuratus) en la zona de Pisco bajo condiciones de El Niño 1983 En: Ibid. Vol. Extraordinario: $87-89$

WOLFF, M. 1988. Spawning and recruitment in the Peruviana Scallop Argopecten purpuratus, Mar Ecol. Prog. Ser., 42: 213 217.

YAMASHIRO, C \& MENDO, J. 1988. Parámetros de crecimiento de la concha de abanico Argopecten purpuratus de la Bahía de In- dependencia, Pisco, Perú. En: Salzwedel, H. y Landa. A. (eds). Recursos y Dinámica del Ecosistema de Afloramiento Peruano. Bol. Inst. Mar Perì-Callao. Vol. Extraordinario 163-168.

YAMASHIRO, C. \& ESPINO, M. 1991. Estimación de la población de concha de abanico (Argopecten purpuratus) mediante la aplicación del método Leslie. En: Comisión Permanente del Pacífico Sur (CPPS). Jordán, S.; Henriquez, C. N. y Kelly, J.R. (eds). Rev. Pacif. Sur, 19: 203-207. 